\title{
Lefschetz properties and basic constructions on simplicial spheres
}

\author{
Eric Babson • Eran Nevo
}

Received: 30 July 2008 / Accepted: 4 June 2009 / Published online: 1 July 2009

(C) Springer Science+Business Media, LLC 2009

\begin{abstract}
The well known $g$-conjecture for homology spheres follows from the stronger conjecture that the face ring over the reals of a homology sphere, modulo a linear system of parameters, admits the strong-Lefschetz property. We prove that the strong-Lefschetz property is preserved under the following constructions on homology spheres: join, connected sum, and stellar subdivisions. The last construction is a step towards proving the $g$-conjecture for piecewise-linear spheres.
\end{abstract}

Keywords Face ring $\cdot$ Strong-Lefschetz property $\cdot$ Homology sphere

\section{Introduction}

Our motivating problem is the following well known $g$-conjecture for spheres, first raised as a question by McMullen for simplicial spheres [15]. By homology sphere we mean a pure simplicial complex $L$ such that for every face $F \in L$ (including the empty set), its $\operatorname{link} \operatorname{lk}(F, L):=\{T \in L: T \cap F=\emptyset, T \cup F \in L\}$ has the same homology (say with integer coefficients) as of a $\operatorname{dim}(\operatorname{lk}(F, L))$-sphere. Any simplicial sphere is a homology sphere.

Conjecture 1.1 (McMullen [15]) The g-vector of any homology sphere is an $M$ sequence, i.e. is the $f$-vector of a multicomplex.

Research of E. Nevo was partially supported by an NSF Award DMS-0757828.

E. Babson

Department of Mathematics, UC Davis, Davis, USA

e-mail: babson@math.ucdavis.edu

E. Nevo (西)

Department of Mathematics, Cornell University, Ithaca, USA

e-mail: eranevo@math.cornell.edu 
An algebraic approach to this problem is to associate with a homology sphere $L$ a standard ring whose Hilbert function is the $g$-vector of $L$. This was worked out successfully by Stanley [22] in his celebrated proof of Conjecture 1.1 for the case where $L$ is the boundary complex of a simplicial polytope. The hard-Lefschetz theorem for toric varieties associated with rational polytopes, translates in this case to the strong-Lefschetz property of face rings, to be defined shortly.

First we introduce some notation. Let $K$ be a $(d-1)$-dimensional simplicial complex on the vertex set $[n]$. Let $\left(\begin{array}{c}{[n]} \\ k\end{array}\right)$ denote the subsets of $[n]$ of size $k$. The $i$-th skeleton of $K$ is $K_{i}=\{S \in K:|S|=i+1\}=K \cap\left(\begin{array}{c}{[n]} \\ i+1\end{array}\right)$, its $f$-vector is $f(K)=$ $\left(f_{-1}, f_{0}, \ldots, f_{d-1}\right)$ where $f_{i}=\left|K_{i}\right|$, its $h$-vector is $h(K)=\left(h_{0}, h_{1}, \ldots, h_{d}\right)$ where $h_{k}=\sum_{0 \leq i \leq k}(-1)^{k-i}\left(\begin{array}{c}d-i \\ k-i\end{array}\right) f_{i-1}$, and in case the $h$-vector is symmetric, its $g$-vector is $g(K)=\left(g_{0}, \ldots, g_{\lfloor d / 2\rfloor}\right)$ where $g_{0}=h_{0}=1$ and $g_{i}=h_{i}-h_{i-1}$ for $1 \leq i \leq\lfloor d / 2\rfloor$.

Let $\mathbb{F}$ be a field, $A=\mathbb{F}\left[x_{1}, . ., x_{n}\right]$ the polynomial ring over $\mathbb{F}$, where each variable has degree one, and $A_{i}$ is the degree $i$ part of $A$. The face ring of $K$, also called Stanley-Reisner ring, is $\mathbb{F}[K]=A / I_{K}$ where $I_{K}$ is the ideal in $A$ generated by the monomials $x^{a}=\Pi_{1 \leq i \leq n} x_{i}^{a_{i}}$ whose support, denoted by $\operatorname{supp}(a)=\left\{i: a_{i}>0\right\}$, is not an element of $K$. Let $\Theta=\left(\theta_{1}, . ., \theta_{d}\right)$ be a linear system of parameters (1.s.o.p. for short) of $\mathbb{F}[K]-$ if $\mathbb{F}$ is infinite it exists, e.g. [23, Lemma 5.2], and generic degree one elements will do. From now on we assume that $\mathbb{F}$ is infinite. Let $H(K)=H(K, \Theta)=$ $\mathbb{F}[K] /(\Theta)=H(K)_{0} \oplus H(K)_{1} \oplus \cdots$ where the grading is induced by the degree grading in $A$, and $(\Theta)$ is the ideal in $\mathbb{F}[K]$ generated by the images of the elements of $\Theta$ under the projection $A \rightarrow \mathbb{F}[K] . K$ is called Cohen-Macaulay (CM for short) over $\mathbb{F}$ if for an (equivalently, every) l.s.o.p. $\Theta, \mathbb{F}[K]$ is a free $\mathbb{F}[\Theta]$-module.

If $K$ is $\mathrm{CM}$ then $\operatorname{dim}_{\mathbb{F}} H(K)_{i}=h_{i}(K)$. (Further, $h$ is an $M$-vector iff $h=h(K)$ for some CM complex $K$ [23, Theorem 3.3].) For $K$ a CM simplicial complex with a symmetric $h$-vector, if there exists an 1.s.o.p. $\Theta$ and an element $\omega \in A_{1}$ such that the multiplication maps $\omega^{d-2 i}: H(K, \Theta)_{i} \longrightarrow H(K, \Theta)_{d-i}, m \mapsto \omega^{d-2 i} m$, are isomorphisms for every $0 \leq i \leq\lfloor d / 2\rfloor$, we say that $K$ has the strong-Lefschetz property, or that $K$ is SL (over $\mathbb{F}$ ). Note that for any complex $K$ the set of $(\Theta, \omega)$ as above is Zariski open (in $\mathbb{F}^{(d+1) n}$ ), but it may be empty. The elements of a specified nonempty Zariski open set are called generic.

As was shown by Stanley [22], for $K$ the boundary complex of a simplicial rational $d$-polytope $P$ with the origin in its interior, the 1.s.o.p $\Theta$ induced by the embedding of its vertices in $\mathbb{R}^{d}$ and $\omega=\sum_{1 \leq i \leq n} x_{i}$ demonstrate that $K$ is SL over $\mathbb{R}$; hence so do generic $(\Theta, \omega)$. Note that if $\mathbb{F}$ is infinite of characteristic zero, then $K$ is SL over $\mathbb{F}$ iff $K$ is SL over $\mathbb{R}$. This follows from writing the SL property as nonvanishing conditions for polynomials in $\mathbb{Z}[\Theta, \omega]$, the polynomial ring with $(d+1) n$ variables over the integers.

Our main result is that the following constructions on homology spheres preserve the strong-Lefschetz property.

Theorem 1.2 Let $K$ and $L$ be homology spheres over an infinite field $\mathbb{F}$, and let $F$ be a face of $K$. Denote by $*$ the join operator, by \# the connected sum operator, and by Stellar $(F, K)$ the stellar subdivision of $K$ at $F$. The following holds:

(1) If $K$ and $L$ are $S L$ over $\mathbb{F}$ and $\mathbb{F}$ has characteristic zero then $K * L$ is an $S L$ homology sphere (over $\mathbb{F}$ ). 
(2) If $K$ and $L$ have the same dimension and are $S L$ over $\mathbb{F}$ then $K \# L$ is an $S L$ homology sphere (over $\mathbb{F})$. (True over any field.)

(3) If $K$ and $\operatorname{lk}(F, K)$ are $S L$ over $\mathbb{F}$ and $\mathbb{F}$ has characteristic zero, then $\operatorname{Stellar}(F, K)$ is an SL homology sphere (over $\mathbb{F}$ ). In particular, if $K$ and all of its face links are $S L$ over $\mathbb{F}$ then the barycentric subdivision of $K$ is $S L$ over $\mathbb{F}$.

Remarks 1.3 (1) Replacing the class of homology spheres by the class of piecewise linear (PL) spheres, Theorem 1.2 still holds. More generally, if $\mathcal{S}$ is a class of simplicial complexes with the SL property, then any complex in its closure w.r.t. join and connected sum is also SL. If $\mathcal{S}$ is closed under links, then any complex in its closure w.r.t. stellar subdivisions is also SL.

(2) Any PL-sphere can be obtained from the boundary of a simplex by a sequence of stellar subdivisions and their inverses (e.g. the survey [14]). Thus, to prove the $g$-conjecture for PL-spheres it is left to prove that the SL property is preserved under the inverse of stellar subdivisions, in the case of PL-spheres.

(3) A result similar to Theorem 1.2(3) was obtained recently, and independently, by Murai [19], using different ideas: if one assumes that $\operatorname{lk}(F, K) * \partial(F \backslash\{u\})$ is SL for some $u \in F$ (instead of that $\operatorname{lk}(F, K)$ is $\operatorname{SL}$ ) then the conclusion that $\operatorname{Stellar}(F, K)$ is SL holds. His proof works for an arbitrary field. Can his proof be used to prove Theorem 1.2(3) for an arbitrary field?

(4) We use Theorem 1.2(1) to prove Theorem 1.2(3). Can Murai's result [19] be used to prove the assertion of Theorem 1.2(1) for an arbitrary field?

The CM property and the strong-Lefschetz property have equivalent formulations in terms of the combinatorics of the symmetric algebraic shifting of the original simplicial complex [10] (definitions and further details appear in Section 3). We consider this reformulation in the context of exterior algebraic shifting, and extend some of our results to this context as well.

This paper is organized as follows: in Section 2 we discuss the effect of join on face rings and prove Theorem 1.2(1). In Section 3 we give background on algebraic shifting and the interpretations of various Lefschetz properties in terms of shifting. In Section 4 we compare the strong and weak-Lefschetz properties, to be used later in the proof of Theorem 1.2(3). In Section 5 we relate a certain Lefschetz type property, in terms of algebraic shifting (symmetric and exterior), to certain edge contractions, and use it to derive Theorem 1.2(3). In Section 6 we show that connected sum preserves both the strong and weak-Lefschetz properties, also in the exterior algebra context; in particular we prove Theorem 1.2(2).

\section{Strong-Lefschetz and join}

The following auxiliary lemma is used in the proof of Theorem 1.2(1).

Lemma 2.1 Let $K$ be a $(d-1)$-dimensional CM complex with a symmetric h-vector, with an l.s.o.p. $\Theta$ and an $S L$ element $\omega$ over $\mathbb{F}$. Let $H=\mathbb{F}[K] /(\Theta)$. Then $H$ decomposes into a direct sum of $\mathbb{F}[\omega]$-modules, each is of the form

$$
V_{m}=\mathbb{F} m \oplus \mathbb{F} \omega m \oplus \cdots \oplus \mathbb{F} \omega^{d-2 i} m
$$


for $m \in \mathbb{F}[K] /(\Theta)$ of degree $i$ for some $0 \leq i \leq d / 2$.

Proof Clearly $V_{1}\left(1 \in H_{0}\right)$ is an $\mathbb{F}[\omega]$-module which contains $H_{0}$. Assume that for $1 \leq i \leq d / 2$ we have already constructed a direct sum of $\mathbb{F}[\omega]$-modules, $\tilde{V}_{i-1}$, which contains $\tilde{H}_{i-1}:=H_{0} \oplus \cdots \oplus H_{i-1}$, in which each $V_{m}$ contains some nonzero element of $\tilde{H}_{i-1}$. We now extend the construction to have these properties w.r.t. $\tilde{H}_{i}$.

Let $W_{i}:=\operatorname{ker}\left(\omega^{d-2 i+1}: H_{i} \rightarrow H_{d-i+1}\right)$, and let $m_{1}, \ldots, m_{t}$ form a basis (over $\mathbb{F}$ ) of $W_{i}$. By the definition of $W_{i}$, each $V_{m_{j}}, 1 \leq j \leq t$, is an $\mathbb{F}[\omega]$-submodule of $H$. As $\omega^{d-2 i}: H_{i} \rightarrow H_{d-i}$ is injective, the sum of the $V_{m_{j}}$ 's is direct, and is denoted by $V_{i}=\bigoplus_{1 \leq j \leq t} V_{m_{j}}$. Let us check that $V_{i} \cap \tilde{V}_{i-1}=0$ by showing that its intersection with each $H_{l}$ is zero. For $l>d-i$ or $l<i$ this is obvious. Otherwise, an element in $V_{i} \cap \tilde{V}_{i-1} \cap H_{l}$ is of the form $\omega^{l-i+1} x=\omega^{l-i} y$ where $x \in H_{i-1}, y \in W_{i}$ and $i \leq l \leq d-i$. As $\omega$ is an SL-element, multiplying by $\omega^{d-i+1-l}$, the LHS stays nonzero while by definition of $W_{i}$ the RHS becomes zero, a contradiction. We now show that the direct sum in degree $i\left(V_{i} \oplus \tilde{V}_{i-1}\right)_{i}$ equals $H_{i}$, by computing dimensions: $\operatorname{dim}_{\mathbb{F}}\left(\tilde{V}_{i-1}\right)_{i}=\operatorname{dim}_{\mathbb{F}}\left(\omega H_{i-1}\right)_{i}=h_{i-1}(K)$, and $\operatorname{dim}_{\mathbb{F}} W_{i}=h_{i}(K)-h_{d-i+1}(K)=h_{i}(K)-h_{i-1}(K)$ hence $\left(V_{i} \oplus \tilde{V}_{i-1}\right)_{i}=H_{i}$ and $\tilde{H}_{i}$ has the desired properties. As the $h$-vector of $K$ is symmetric, $H=\tilde{H}_{\lfloor d / 2\rfloor}$, which completes the proof.

Recall that the join of two simplicial complexes with disjoint sets of vertices is $K * L:=\{S \cup T: S \in K, T \in L\}$.

Theorem 2.2 Let $K$ and $L$ be CM complexes over an infinite field $\mathbb{F}$ on disjoint sets of vertices, with symmetric h-vectors, of dimensions $d_{K}-1, d_{L}-1$, with l.s.o.p's $\Theta_{K}, \Theta_{L}$ and SL elements $\omega_{K}, \omega_{L}$ respectively; over $\mathbb{F}$. Then:

(0) $K * L$ is a CM complex of dimension $d_{K}+d_{L}-1$ with a symmetric $h$-vector.

(1) $\Theta_{K} \biguplus \Theta_{L}$ is an l.s.o.p for $K * L$ (over $\left.\mathbb{F}\right)$.

(2) If char $(\mathbb{F})=0$ then $\omega_{K}+\omega_{L}$ is an SL element of $\mathbb{F}[K * L] /\left(\Theta_{K} \biguplus \Theta_{L}\right)$.

We thank one of the referees for pointing out to us the relevance of [6]. Theorem 2.2 easily follows from [6, Theorem 11]; we leave the proof below for the sake of completeness.

Proof (0) is easy and well known. A topological way to see that $K * L$ is $\mathrm{CM}$ is to use Reisner theorem and Künneth theorem. An algebraic way will be described in the proof of (1). To see that $K * L$ has a symmetric $h$-vector note that the product of symmetric polynomials is a symmetric polynomial.

We now exhibit a special l.s.o.p. for $K * L$. For a set $I$ let $A_{I}:=\mathbb{F}\left[x_{i}: i \in I\right]$ be a polynomial ring. The isomorphism

$$
A_{K_{0}} \bigotimes_{\mathbb{F}} A_{L_{0}} \cong A_{K_{0} \biguplus L_{0}}, a_{K} \otimes a_{L} \mapsto a_{K} a_{L}
$$

induces a structure of an $A=A_{K_{0} \biguplus L_{0}}$ module on $\mathbb{F}[K] \bigotimes_{\mathbb{F}} \mathbb{F}[L]$, isomorphic to $\mathbb{F}[K * L]$, by $m_{K} \otimes m_{L} \mapsto m_{K} m_{L}$ and $\left(a_{K} \otimes a_{L}\right)\left(m_{K} \otimes m_{L}\right)=a_{K} m_{K} \otimes a_{L} m_{L}$. (E.g. $a_{K} \in A_{K_{0}} \subseteq A$ acts like $a_{K} \otimes 1$ on $\mathbb{F}[K] \bigotimes_{\mathbb{F}} \mathbb{F}[L]$. ) 
The above isomorphism induces an isomorphism of $A$-modules

$$
\mathbb{F}[K * L] /\left(\Theta_{K} \biguplus \Theta_{L}\right) \mathbb{F}[K * L] \cong \mathbb{F}[K] /\left(\Theta_{K}\right) \mathbb{F}[K] \bigotimes_{\mathbb{F}} \mathbb{F}[L] /\left(\Theta_{L}\right) \mathbb{F}[L],
$$

proving part (1).

By Lemma $2.1, \mathbb{F}[K] /\left(\Theta_{K}\right)$ decomposes into a direct sum of $\mathbb{F}\left[\omega_{K}\right]$-modules, each of the form $V_{m}(K)=\mathbb{F} m \bigoplus \mathbb{F} \omega_{K} m \bigoplus \cdots \bigoplus \mathbb{F} \omega_{K}^{d_{K}-2 i} m$ for $m \in \mathbb{F}[K] /\left(\Theta_{K}\right)$ of degree $i$ for some $0 \leq i \leq d_{K} / 2$; and similarly for $\mathbb{F}[L] /\left(\Theta_{L}\right)$.

As $\mathbb{F}$ is infinite of characteristic zero, to prove (2) we may assume $\mathbb{F}=\mathbb{R}$. The $\mathbb{R}\left[\omega_{K}\right]$-module $V_{m}(K)$ is isomorphic to the $\mathbb{R}[\omega]$-module $\mathbb{R}\left[\partial \sigma^{d_{K}-2 i}\right] /(\theta)$ by $\omega_{K} \mapsto \omega$ and $m \mapsto 1$, where $\sigma^{j}$ is the $j$-simplex, $\theta$ is an 1.s.o.p. induced by the positions of the vertices in an embedding of $\sigma^{d_{K}-2 i}$ as a full dimensional geometric simplex in $\mathbb{R}^{d_{K}-2 i}$ with the origin in its interior, and $\omega=\sum_{v \in \sigma_{0}} x_{v}$ is an SL element for $\mathbb{R}\left[\partial \sigma^{d_{K}-2 i}\right] /(\theta)$. By equation (1), $\mathbb{R}[K * L] /\left(\Theta_{K} \biguplus \Theta_{L}\right)$ is isomorphic as an $\mathbb{R}\left[\omega_{K}+\omega_{L}\right]$-module to a direct sum of submodules of the form $V_{m}(K) \otimes V_{m^{\prime}}(L)$. To prove part (2), we will show that $\omega_{K}+\omega_{L}$ is an SL element of each direct summand. Thus, it is enough to prove it for the join of boundaries of two simplices with 1.s.o.p.'s as above and the SL elements having weight 1 on each vertex of the ground set.

Note that the join $\partial \sigma^{k} * \partial \sigma^{l}$ is combinatorially isomorphic to the boundary of the polytope $P:=\operatorname{conv}\left(\sigma^{k} \cup \sigma^{l}\right)$ where $\sigma^{k}$ and $\sigma^{l}$ are embedded in orthogonal spaces and intersect only in the origin which is in the relative interior of both. McMullen's proof of the $g$-theorem for simplicial polytopes [16, 17] states that $\sum_{v \in P_{0}} x_{v}=\omega_{\partial \sigma^{k}}+\omega_{\partial \sigma^{l}}$ is indeed an SL element of $\mathbb{R}\left[\partial \sigma^{k} * \partial \sigma^{l}\right] /\left(\Theta_{\partial P}\right)$ where $\Theta_{\partial P}$ is the 1.s.o.p. induced by the positions of the vertices in the polytope $P$. By the definition of $P, \Theta_{\partial P}=\Theta_{\partial \sigma^{k}} \uplus \Theta_{\partial \sigma^{l}}$. Thus part (2) is proved.

In particular, Theorem 2.2 implies Theorem 1.2(1). Similarly, as the join of PL spheres is a PL sphere, Remark 1.3(1) follows in the same manner.

Remarks 2.3 (1) As a nonzero multiple of an SL element is again SL, in Theorem 2.2(2) any element $a \omega_{K}+b \omega_{L}$ where $a, b \in \mathbb{F}, a b \neq 0$, will do.

(2) The case $\operatorname{char}(\mathbb{F}) \neq 0$ : the proof above shows that for $\omega=\omega_{K}+\omega_{L}$ we get an isomorphism of $\mathbb{F}[\omega]$-modules

$$
V_{m(K)} \bigotimes_{\mathbb{F}} V_{m(L)} \cong \frac{\mathbb{F}\left[\omega_{K}\right]}{\left(\omega_{K}^{d_{K}-2 i_{K}+1}\right) \mathbb{F}\left[\omega_{K}\right]} \bigotimes_{\mathbb{F}} \frac{\mathbb{F}\left[\omega_{L}\right]}{\left(\omega_{L}^{d_{L}-2 i_{L}+1}\right) \mathbb{F}\left[\omega_{L}\right]}
$$

over any infinite field $\mathbb{F}$. Picking the basis $\left\{\omega_{K}^{l} \otimes \omega_{L}^{j}: 0 \leq l \leq d_{K}-2 i_{K}, 0 \leq j \leq\right.$ $\left.d_{L}-2 i_{L}\right\}$ for the module on the RHS, we see that the representing matrix of the $\operatorname{map} \omega^{d_{K}+d_{L}-2 i}:\left(V_{m(K)} \otimes V_{m(L)}\right)_{i} \rightarrow\left(V_{m(K)} \otimes V_{m(L)}\right)_{d_{K}+d_{L}-i}$ consists of integer entries (all entries are binomials). However, if $\operatorname{char}(\mathbb{F}) \neq 0$, the determinant of this matrix may equal zero. In other words, there exist simplices $\sigma^{d_{K}}, \sigma^{d_{L}}$ such that for any 1.s.o.p's $\Theta_{K}, \Theta_{L}$ of the face rings of their boundaries, respectively, there is no SLelement for $\mathbb{F}\left[\partial \sigma^{d_{K}} * \partial \sigma^{d_{L}}\right] /\left(\Theta_{K} \cup \Theta_{L}\right)$. On the other hand, for strongly edge decomposable complexes, introduced in [21], Murai proved recently, see [19, Corollary 3.5], 
that the SL property holds over any field. The join of boundaries of two simplices is strongly edge decomposable (identify a pair of vertices, one from each simplex, to obtain the boundary of a simplex), hence for some other 1.s.o.p $\Theta, \mathbb{F}\left[\partial \sigma^{d_{K}} * \partial \sigma^{d_{L}}\right] /(\Theta)$ has an SL-element. This raises the following question:

Problem 2.4 Does Theorem 1.2(1) hold for a field of an arbitrary characteristic? Can the results in [19] be used to prove this?

\section{Algebraic shifting}

Let $<$ denote the usual order on the natural numbers. A simplicial complex $K$ with vertices $[n]=\{1,2, \ldots, n\}$ is shifted if for every $i<j$ and $j \in S \in K$, also $(S \backslash\{j\}) \cup$ $\{i\} \in K$.

Algebraic shifting is an operator associating with each simplicial complex a shifted simplicial complex. It has two versions - exterior and symmetric, both introduced by Kalai. Various invariants of the original complex, like its $f$-vector and Betti numbers, can be read off from its shifting. For a survey on algebraic shifting see Kalai [11]. For completeness we give now the definitions of exterior and symmetric shifting.

Exterior shifting. Let $\mathbb{F}$ be an infinite field. Let $V$ be an $n$-dimensional vector space over $\mathbb{F}$ with basis $\left\{e_{1}, \ldots, e_{n}\right\}$. Let $\wedge V$ be the graded exterior algebra over $V$. Let $e_{S}=e_{s_{1}} \wedge \cdots \wedge e_{s_{j}}$ where $S=\left\{s_{1}<\cdots<s_{j}\right\}$. Then $\left\{e_{S}: S \in\left(\begin{array}{c}{[n]} \\ j\end{array}\right)\right\}$ is a basis for $\bigwedge^{j} V$. Note that as $K$ is a simplicial complex, the ideal $\left(e_{S}: S \notin K\right)$ of $\wedge V$ and the vector subspace $\operatorname{span}\left\{e_{S}: S \notin K\right\}$ of $\bigwedge V$ consist of the same set of elements in $\bigwedge V$. Define the exterior algebra of $K$ by

$$
\bigwedge K=(\bigwedge V) /\left(e_{S}: S \notin K\right)
$$

Let $\left\{f_{1}, \ldots, f_{n}\right\}$ be a basis of $V$ with a corresponding transition matrix $A\left(e_{i} A=f_{i}\right.$ for all $i$ ). Let $\tilde{f}_{S}$ be the image of $f_{S}=f_{s_{1}} \wedge \cdots \wedge f_{s_{j}} \in \wedge V$ in $\wedge K$, where $S=$ $\left\{s_{1}<\cdots<s_{j}\right\}$. Let $<_{L}$ be the lexicographic order on equal sized subsets of $\mathbb{N}$, i.e. $S<_{L} T$ iff $\min (S \triangle T) \in S$, where $\triangle$ denotes symmetric difference. Define

$$
\Delta_{A}^{e}(K)=\left\{S: \tilde{f}_{S} \notin \operatorname{span}\left\{\tilde{f}_{S^{\prime}}: S^{\prime}<_{L} S\right\}\right\} .
$$

Then there is a nonempty Zariski open set $U \subseteq \mathbb{F}^{n^{2}}$ such that $\Delta_{A}^{e}(K)$ is the same for all $A \in U$. This complex was introduced by Kalai [7] and is called the exterior shifting of $K$, denoted by $\Delta^{e}(K)$. Indeed it is well defined as two nonempty Zariski open sets must intersect. (Kalai used a field extension of $\mathbb{F}$ in his definition, and let the entries of $A$ be algebraically independent over $\mathbb{F}$. However the two definitions are equivalent.)

The construction is canonical, i.e. it is independent of the choice of the generic matrix $A$, and for a permutation $\pi:[n] \rightarrow[n]$ the induced simplicial complex $\pi(K)$ satisfies $\Delta^{e}(\pi(K))=\Delta^{e}(K)$. It results in a shifted simplicial complex, having the same face vector and Betti vector as $K[2]$. 
Symmetric shifting. Let $\mathbb{F}$ be an infinite field, and $\mathbb{F}[K]=\mathbb{F}\left[x_{1}, \ldots, x_{n}\right] / I_{K}$ the face ring (Stanley-Reisner ring) of $K$, i.e. $I_{K}$ is the homogeneous ideal generated by the squarefree monomials whose support is not in $K,\left\{\prod_{i \in S} x_{i}: S \notin K\right\} . \mathbb{F}[K]$ is graded by degree. Let $y_{1}, \ldots, y_{n}$ be generic linear combinations of $x_{1}, \ldots, x_{n}$. We choose a basis for each graded component of $\mathbb{F}[K]$, up to degree $\operatorname{dim}(K)+1$, from the canonical projection of the monomials in the $y_{i}$ 's on $\mathbb{F}[K]$, in the greedy way:

$$
\operatorname{GIN}(K)=\left\{m: \tilde{m} \notin \operatorname{span}_{\mathbb{F}}\left\{\tilde{m^{\prime}}: \operatorname{deg}\left(m^{\prime}\right)=\operatorname{deg}(m), m^{\prime}<_{L} m\right\}\right\}
$$

where $\prod y_{i}^{a_{i}}<_{L} \prod y_{i}^{b_{i}}$ iff for $j=\min \left\{i: a_{i} \neq b_{i}\right\} a_{j}>b_{j}$. The combinatorial information carried by $\operatorname{GIN}(K)$ is redundant: if $m \in \operatorname{GIN}(K)$ is of degree $i \leq \operatorname{dim}(K)$ then $y_{1} m, \ldots, y_{i} m$ are also in $\operatorname{GIN}(K)$. Thus, $\operatorname{GIN}(K)$ can be reconstructed from its monomials of the form $m=y_{i_{1}} \cdot y_{i_{2}} \cdot \ldots \cdot y_{i_{r}}$ where $r \leq i_{1} \leq i_{2} \leq \ldots \leq i_{r}$, $r \leq \operatorname{dim}(K)+1$. Denote this set by $\operatorname{gin}(K)$, and define $S(m)=\left\{i_{1}-r+1, i_{2}-\right.$ $\left.r+2, \ldots, i_{r}\right\}$ for such $m$. The collection of sets

$$
\Delta^{s}(K)=\{S(m): m \in \operatorname{gin}(K)\}
$$

carries the same combinatorial information as $\operatorname{GIN}(K) . \Delta^{s}(K)$ is a simplicial complex. Again, the construction is canonical, in the same sense as for exterior shifting. If $\mathbb{F}$ has characteristic zero then $\Delta^{s}(K)$ is shifted [9].

Lefschetz properties via shifting. $K$ is CM (over $\mathbb{F}$ ) iff $\Delta^{s}(K)$ is pure (i.e. all its maximal faces have the same size) and the following condition holds

$$
S \in \Delta^{s}(K),|S|=k \Rightarrow[d-k] \cup S \in \Delta^{s}(K) .
$$

To see this take the first $d$ elements in a generic basis, $\left\{y_{1}, \ldots, y_{d}\right\}$, to be an 1.s.o.p. for $K$.

Further, let $\Delta(d, n)$ be the pure $(d-1)$-dimensional simplicial complex with the vertex set $[n]$ and facets

$$
\{S: S \subseteq[n],|S|=d, k \notin S \Rightarrow[k+1, d-k+2] \subseteq S\} .
$$

Equivalently, $\Delta(d, n)$ is the maximal pure $(d-1)$-dimensional simplicial complex with vertex set $[n]$ which does not contain any of the sets $T_{d}^{d}, \ldots, T_{\lceil d / 2\rceil}^{d}$, where

$$
T_{d-k}^{d}=\{k+2, k+3, \ldots, d-k, d-k+2, d-k+3, \ldots, d+2\}, 0 \leq k \leq\lfloor d / 2\rfloor .
$$

Note that $\Delta(d, n) \subseteq \Delta(d, n+1)$, and define $\Delta(d)=\cup_{n} \Delta(d, n)$. For $K$ a CM $(d-1)$-dimensional complex with symmetric $h$-vector, $\Delta^{s}(K) \subseteq \Delta(d)$ is equivalent to $K$ being SL. To see this, take the $(d+1)$ 'th element in a generic basis, $y_{d+1}$, to be the strong-Lefschetz element: indeed, $\Delta^{s}(K) \subseteq \Delta(d)$ iff none of the monomials $y_{d+1}^{d-2 k-1} y_{d+2}^{k+1}$ are in $\operatorname{GIN}(K)$ (where $k=0,1, \ldots$ ), which happens iff the maps $y_{d+1}^{d-2 k}: H(K)_{k} \longrightarrow H(K)_{d-k}$ are onto for $0 \leq k \leq\lfloor d / 2\rfloor$, and when $h(K)$ is symmetric this happens iff these maps are isomorphisms.

Let $\Delta(K)$ refer to both symmetric and exterior shifting. Kalai refers to the relation

$$
\Delta(K) \subseteq \Delta(d)
$$


as the shifting theoretic upper bound theorem. To justify the name, note that the boundary complex of the cyclic $d$-polytope on $n$ vertices, denoted by $C(d, n)$, satisfies $\Delta^{s}(C(d, n))=\Delta(d, n)$. This follows from the fact that $C(d, n)$ is SL. Recently Murai [18] proved that also $\Delta^{e}(C(d, n))=\Delta(d, n)$, as was conjectured by Kalai [11]. It follows that if $K$ has $n$ vertices and (4) holds, then the $f$-vectors satisfy $f(K) \leq f(C(d, n))$ componentwise.

For $K$ as above (CM with symmetric $h$-vector), a condition weaker than the strong-Lefschetz property is to require only that multiplications $y_{d+1}: H(K)_{i-1} \longrightarrow$ $H(K)_{i}$ are either injective (for $1 \leq i \leq\lceil d / 2\rceil$ ) or surjective (for $\lceil d / 2\rceil<i \leq d$ ). This condition is usually called in the literature the weak-Lefschetz property (WL for short). Even weaker condition is just to require that multiplications $y_{d+1}$ : $H(K)_{i-1} \longrightarrow H(K)_{i}$ are injective for $1 \leq i \leq\lfloor d / 2\rfloor$, called here WWL property. (Injectivity for $i \leq\lceil d / 2\rceil$ in the case of homology spheres implies also surjective maps for $\lceil d / 2\rceil<i \leq d$ as was noticed by Swartz; see the proof of Theorem 4.2 below.) The WWL property is equivalent to the following, in the case of symmetric shifting [3]:

$$
\begin{array}{r}
S \in \Delta(K),|S|=k \Rightarrow[d-k] \cup S \in \Delta(K), \\
S \in \Delta(K),|S|=k<\lfloor d / 2\rfloor \Rightarrow\{d-k+1\} \cup S \in \Delta(K) .
\end{array}
$$

The first condition holds when $K$ is CM, and the second condition holds iff $K$ is WWL. As was noticed in [3], (5) is implied by requiring that $\Delta(K)$ is pure and every $S \in \Delta(K)$ of size less than $\lfloor d / 2\rfloor$ is contained in at least 2 facets of $\Delta(K)$.

Note that if $L$ is a homology sphere, it is in particular CM with a symmetric $h$-vector. If in addition it is WWL, then in the standard ring $S(L)=$ $\mathbb{F}[L] /\left(y_{1}, \ldots, y_{d+1}\right)=H\left(L,\left\{y_{1}, \ldots, y_{d}\right\}\right) /\left(y_{d+1}\right)=S_{0} \oplus S_{1} \oplus \ldots$ the following holds: $g_{i}(L)=\operatorname{dim}_{\mathbb{F}} S_{i}$ for all $0 \leq i \leq\lfloor d / 2\rfloor$, and Conjecture 1.1 holds for $L$.

We summarize the discussion above in the following hierarchy of conjectures, where assertion $(i)$ implies assertion $(i+1)$ :

Conjecture 3.1 Let L be a homology $(d-1)$-sphere. Then:

(1) If $S \in \Delta(L),|S|=k \leq\lfloor d / 2\rfloor$ and $S \cap[d-k+1]=\emptyset$ then $S \cup[k+2, d-$ $k+1] \in \Delta(L)$.

This is equivalent to $\Delta(K) \subseteq \Delta(d)$, and in the symmetric case this is equivalent to $L$ being $S L$.

(2) If $S \in \Delta(L),|S|=k<\lfloor d / 2\rfloor$ and $S \cap[d-k+1]=\emptyset$ then $S \cup[\lceil d / 2\rceil+2, d-$ $k+1] \in \Delta(L)$. In the symmetric case this is equivalent to $L$ being $W W L$.

(3) $g(L)$ is an $M$-vector.

To see that the conclusion of Conjecture 3.1(1) is equivalent to equation (4) use the fact that $\Delta(K)$ is pure and shifted. The equivalence of the conclusion of Conjecture 3.1(2) and equation (5) is obvious.

\section{Strong Lefschetz versus weak-Lefschetz}

Examples of Gorenstein algebras admitting the weak-Lefschetz property but not the strong-Lefschetz property were found in [5, Example 4.3]. For Gorenstein algebras 
arising as face rings of homology spheres the SL property is conjectured to hold. Does it follow from the (conjectured) WL property for homology spheres? We end this section with a result in this direction, to be used later in the proof of Theorem 1.2(3).

Consider the multiplication maps $\omega_{i}: H(K, \Theta)_{i} \longrightarrow H(K, \Theta)_{i+1}, m \mapsto \omega_{i} m$ where $\omega_{i} \in A_{1}$. Let $\operatorname{dim}(K)=d-1$. Denote by $\Omega_{W L}(K, i)$ the set of all $\left(\Theta, \omega_{i}\right) \in$ $A_{1}^{d+1}$ such that $\Theta$ is an 1.s.o.p. of $\mathbb{F}[K], \mathbb{F}[K]$ is a free $\mathbb{F}[\Theta]$-module, and $\omega_{i}$ : $H(K)_{i} \longrightarrow H(K)_{i+1}$ is either injective (and $i<d / 2$ ) or surjective (and $i \geq d / 2$ ). Denote by $\Omega_{S L}(K, i)$ the set of all $(\Theta, \omega) \in A_{1}^{d+1}$ such that $\Theta$ is an 1.s.o.p. of $\mathbb{F}[K], \mathbb{F}[K]$ is a free $\mathbb{F}[\Theta]$-module, and $\omega^{d-2 i}: H(K)_{i} \longrightarrow H(K)_{d-i}$ is injective $(0 \leq i \leq\lfloor d / 2\rfloor)$. If $\Omega_{S L}(K, i) \neq \emptyset$ we say that $K$ is $i$-Lefschetz and for $(\Theta, \omega) \in$ $\Omega_{S L}(K, i)$ that $H(K, \Theta)$ is $i$-Lefschetz with an $i$-Lefschetz element $\omega$. For $d$ odd $\Omega_{W L}(K,\lfloor d / 2\rfloor)=\Omega_{S L}(K,\lfloor d / 2\rfloor)$, which we simply denote by $\Omega(K,\lfloor d / 2\rfloor)$.

The following is well known, see e.g. [24, Proposition 3.6] for the case $\Omega_{S L}(K, i)$; similar arguments can be used to prove the same conclusion for $\Omega_{W L}(K, i)$.

Lemma 4.1 For every simplicial complex $K$ and for every $i, \Omega_{W L}(K, i)$ is a Zariski open set. For $0 \leq i \leq\left\lfloor\frac{\operatorname{dim}(K)+1}{2}\right\rfloor, \Omega_{S L}(K, i)$ is a Zariski open set. (They may be empty, e.g. if $K$ is not pure.)

Theorem 4.2 (Swartz) Let $d \geq 1$. If for every $0 \leq m \leq d$ and every homology $2 m$ sphere $L, \Omega(L, m)$ is nonempty, then for every $t>2 d$ and for every homology $t$ sphere $K, \Omega_{W L}(K, m)$ is nonempty for every $0 \leq m \leq d$. In particular, if for every even dimensional homology sphere $L, \Omega\left(L, \frac{\operatorname{dim}(L)}{2}\right) \neq \emptyset$ then Conjecture 1.1 follows.

Proof By [25, Theorem 4.26] and induction on $t$, for any $0 \leq m \leq d, \Omega_{W L}(K$, $(t+1)-(m+1))$ is nonempty, i.e. multiplication $\omega: H(K)_{t-m} \rightarrow H(K)_{t-m+1}$ is surjective for a generic 1.s.o.p. and $\omega$ in $A_{1}$. Hence, for the canonical module $\Omega(K)$, multiplication by a generic degree 1 element $\omega:(\Omega(K) / \Theta \Omega(K))_{m} \rightarrow$ $(\Omega(K) / \Theta \Omega(K))_{m+1}$ is injective in the first $d$ degrees. As $K$ is a homology sphere, $\Omega(K) \cong \mathbb{R}[K]$ as graded $A$-modules up to a shift in grading (e.g. [23]), hence $\Omega_{W L}(K, m)$ is nonempty for every $m \leq d$. Combining this with Lemma 4.1 , and the fact that a finite intersection of Zariski nonempty open sets is nonempty, we obtain that if the conditions of Theorem 4.2 are met for every $d \geq 1$ then every homology sphere is WL, and hence Conjecture 1.1 follows.

We wish to show further, that if all even dimensional homology spheres satisfy the condition in Theorem 4.2 then all homology spheres are SL. The following result aims at this direction. If one could extend the conclusion of Lemma 4.3 below for every 1.s.o.p. of $S * \partial \sigma$ (not only of the form $\Theta_{S} \cup \Theta_{\partial \sigma}$ ), then indeed WL would imply SL for homology spheres. Compare with [6, Proposition 19].

Lemma 4.3 Let $S$ be a homology sphere with an l.s.o.p. $\Theta_{S}$ over a field $\mathbb{F}$ of characteristic zero. If $H\left(S, \Theta_{S}\right)$ is $\left(\left\lfloor\frac{\operatorname{dim} S+1}{2}\right\rfloor\right)$-Lefschetz but not SL then there exists a simplex $\sigma$ such that the homology sphere $S * \partial \sigma$ is of even dimension $2 j$, and for every l.s.o.p. $\Theta_{\partial \sigma}$ of $\partial \sigma, \mathbb{F}[S * \partial \sigma] /\left(\Theta_{S} \cup \Theta_{\partial \sigma}\right)$ has no $j$-Lefschetz element; in particular $\mathbb{F}[S * \partial \sigma] /\left(\Theta_{S} \cup \Theta_{\partial \sigma}\right)$ is not $W L$. 
Proof Denote the dimension of $S$ by $d-1$ and recall that $A_{S_{0}}=\mathbb{F}\left[x_{v}: v \in S_{0}\right]$. By Lemma $4.1 \Omega_{S L}(S, i)$ is a Zariski open set for every $0 \leq i \leq\lfloor d / 2\rfloor$. The assumption that $S$ is not SL (but is ( $\left\lfloor\frac{d}{2}\right\rfloor$ )-Lefschetz) implies that there exists $0 \leq i_{0} \leq\lfloor d / 2\rfloor-1$ such that $\Omega_{S L}\left(S, i_{0}\right)=\emptyset$ (as a finite intersection of Zariski nonempty open sets is nonempty). Hence, for the fixed l.s.o.p. $\Theta_{S}$ and every $\omega_{S} \in\left(A_{S_{0}}\right)_{1}$, there exists $0 \neq$ $m=m\left(\omega_{S}\right) \in H_{i_{0}}(S)$ such that $\omega_{S}^{d-2 i_{0}} m=0$.

Let $T=S * \partial \sigma$ where $\sigma$ is the $\left(d-2 i_{0}-1\right)$-simplex. Note that $\operatorname{dim}(\sigma) \geq 1$, hence $\partial \sigma \neq \emptyset$. Then $T$ is a homology sphere of even dimension $2 d-2 i_{0}-2$. We have seen (Theorem 2.2) that for any 1.s.o.p. $\Theta_{\partial \sigma}$ of $\partial \sigma, \Theta_{T}:=\Theta_{S} \cup \Theta_{\partial \sigma}$ is an 1.s.o.p. of $T$. Every $\omega_{T} \in\left(A_{T_{0}}\right)_{1}$ has a unique expansion $\omega_{T}=\omega_{S}+\omega_{\partial \sigma}$ where $\omega_{S} \in\left(A_{S_{0}}\right)_{1}$ and $\omega_{\partial \sigma} \in\left(A_{\partial \sigma_{0}}\right)_{1}$. Recall the isomorphism (1) of $A_{T_{0}}$-modules $\mathbb{F}[T] /\left(\Theta_{T}\right) \cong \mathbb{F}[S] /\left(\Theta_{S}\right) \otimes_{\mathbb{F}} \mathbb{F}[\partial \sigma] /\left(\Theta_{\partial \sigma}\right)$. Let $m\left(\omega_{T}\right) \in\left(\frac{\mathbb{F}[T]}{\left(\Theta_{T}\right)}\right)_{d-i_{0}-1}$ be

$$
m\left(\omega_{T}\right):=\sum_{0 \leq j \leq d-2 i_{0}-1}(-1)^{j} \omega_{S}^{d-2 i_{0}-1-j} m \otimes \omega_{\partial \sigma}^{j} .
$$

Note that the sum $\omega_{T} m\left(\omega_{T}\right)$ is telescopic, thus $\omega_{T} m\left(\omega_{T}\right)=\omega_{S}^{d-2 i_{0}} m \otimes 1+$ $(-1)^{d-2 i_{0}-1} m \otimes \omega_{\partial \sigma}^{d-2 i_{0}} 1=0+0=0$. For a generic $\omega_{T}$, the projection of $\omega_{\partial \sigma}$ on $\mathbb{F}[\partial \sigma] /\left(\Theta_{\partial \sigma}\right)$ is nonzero, hence so is the projection of $\omega_{\partial \sigma}^{d-2 i_{0}-1}$, and we get that $m\left(\omega_{T}\right) \neq 0$. Thus, Zariski topology tells us that for every $\omega_{T} \in\left(A_{T_{0}}\right)_{1}$, there exists $0 \neq m\left(\omega_{T}\right) \in\left(\frac{\mathbb{F}[T]}{\left(\Theta_{T}\right)}\right)_{d-i_{0}-1}$ such that $\omega_{T} m\left(\omega_{T}\right)=0$.

\section{Lefschetz properties and Stellar subdivisions}

Roughly speaking, we will show that Stellar subdivisions preserve the SL property when $\mathbb{F}$ has characteristic zero. As mentioned in the Introduction, we may assume $\mathbb{F}=\mathbb{R}$.

Proposition 5.1 Let $K$ be a simplicial complex. Let $K^{\prime}$ be obtained from $K$ by identifying two distinct vertices $u$ and $v$ in $K$, i.e. $K^{\prime}=\{T: u \notin T \in K\} \cup\{(T \backslash\{u\}) \cup\{v\}$ : $u \in T \in K\}$. Let $d \geq 2$. Assume that $\{d+2, d+3, \ldots, 2 d+1\} \notin \Delta\left(K^{\prime}\right)$ and that $\{d+1, d+2, \ldots, 2 d-1\} \notin \Delta(\operatorname{lk}(u, K) \cap \operatorname{lk}(v, K))$. Then $\{d+2, d+3, \ldots, 2 d+1\} \notin$ $\Delta(K)$. (Shifting is over $\mathbb{R}$ for $\Delta^{s}$ and over any infinite field for $\Delta^{e}$.)

The case $d=2$ and $\operatorname{dim}(K)=1$ of this proposition was proved by Whiteley [27] in the symmetric case. The relation between symmetric shifting and rigidity of graphs, discussed in Lee [13], is used to translate his result to algebraic shifting terms.

Proof for symmetric shifting Let $\psi: K_{0} \longrightarrow \mathbb{R}^{2 d}$ be generic (in the space $\mathbb{R}^{2 d\left|K_{0}\right|}$ ). Then $\psi$ represents a tuple of $2 d$ linear forms taken from a suitable nonempty Zariski open set (it includes e.g. all $\psi$ such that the entries of the representing matrix w.r.t. a fixed basis are algebraically independent over $\mathbb{Q}$ ). It induces the following map: 


$$
\begin{aligned}
\psi_{K}^{2 d}: \oplus_{T \in K_{d-1}} \mathbb{R} T & \longrightarrow \oplus_{F \in\left(\begin{array}{c}
K_{0} \\
d-1
\end{array}\right)} \mathbb{R}^{2 d} / \operatorname{span}(\psi(F)), \\
1 T & \mapsto \sum_{F \in\left(\begin{array}{c}
K_{0} \\
d-1
\end{array}\right)} \delta_{F \subseteq T} \overline{\psi(T \backslash F)} F
\end{aligned}
$$

where $\delta_{F \subseteq T}$ equals 1 if $F \subseteq T$ and 0 otherwise, and $\bar{a}$ in the $F$-coordinate denotes the image of $a$ in $\mathbb{R}^{2 d} / \operatorname{span}(\psi(F))$. Thus the image of $\psi_{K}^{2 d}$ is in the tautological vector bundle, denoted by $\tau_{K, d}$, over the Grassmannian $\operatorname{Gr}\left((\mathrm{d}-1)\left(\begin{array}{l}\left|\mathrm{K}_{0}\right| \\ \mathrm{d}-1\end{array}\right), \mathbb{R}^{2 \mathrm{~d}\left(\begin{array}{l}\left|\mathrm{K}_{0}\right| \\ \mathrm{d}-1\end{array}\right)}\right)$.

Recall that $\{d+2, d+3, \ldots, 2 d+1\} \notin \Delta^{s}(K)$ iff $y_{2 d+1}^{d} \notin \operatorname{GIN}(K)$, where $Y=$ $\left\{y_{i}\right\}_{i \in K_{0}}$ is a generic basis for $A_{1}$ and $A=\mathbb{R}\left[x_{v}: v \in K_{0}\right]$. By Lee [13, Theorems $10,12,15]$ and Tay, White and Whiteley [26, Proposition 5.2], $y_{2 d+1}^{d} \notin \operatorname{GIN}(K)$ iff $\operatorname{Ker} \phi_{K}^{2 d}=0$ for some $\phi: K_{0} \longrightarrow \mathbb{R}^{2 d}$ (equivalently, every $\phi$ in some Zariski nonempty open set of maps).

Consider the following degenerating map: for $0<t \leq 1$ let $\psi_{t}: K_{0} \longrightarrow \mathbb{R}^{2 d}$ be defined by $\psi_{t}(i)=\psi(i)$ for every $i \neq u$ and $\psi_{t}(u)=\psi(v)+t(\psi(u)-\psi(v))$. Let $\psi_{0}=\lim _{t \mapsto 0} \psi_{t}$. Thus $\psi_{1}=\psi$, and for any $0<t \leq 1$,

$$
\operatorname{span}\left(\psi_{t}(u)-\psi_{t}(v)\right)=\operatorname{span}(\psi(u)-\psi(v))
$$

hence the same equality holds in the limit $t \mapsto 0$.

Let $\psi_{K, t}^{2 d}: \oplus_{T \in K_{d-1}} \mathbb{R} T \longrightarrow \oplus_{F \in\left(\begin{array}{c}K_{0} \\ d-1\end{array}\right)} \mathbb{R}^{2 d} / \operatorname{span}\left(\psi_{t}(F)\right)$ be the map induced by $\psi_{t}$. Thus $\psi_{K, 1}^{2 d}=\psi_{K}^{2 d}$, and for $\{u, v\} \subseteq F \in\left(\begin{array}{c}K_{0} \\ d-1\end{array}\right), \lim _{t \mapsto 0} \operatorname{span}\left(\psi_{t}(F)\right)=$ $\operatorname{span}(\psi(F))$.

Let $\psi_{0}^{2 d}$ be the limit map $\lim _{t \mapsto 0} \psi_{K, t}^{2 d}$. This limit, which we describe explicitly below, is then the (obviously unique) continuous extension of $\psi_{K,}^{2 d}: \mathbb{R} K_{d-1} \times(0,1] \rightarrow$ $\tau_{K, d}$ to $\mathbb{R} K_{d-1} \times[0,1]$, where $\psi_{K, .}^{2 d}(s, t)=\psi_{K, t}^{2 d}(s)$ for $0<t \leq 1$. Let $\left.a\right|_{F}$ denote the coefficient vector of $a$ in the base coordinate $F$. Then $\psi_{0}^{2 d}$ reads as follows, where $T \in K_{d-1}$ and $F \in\left(\begin{array}{c}K_{0} \\ d-1\end{array}\right)$ :

$$
\begin{aligned}
& \left.\psi_{0}^{2 d}(1 T)\right|_{F} \\
& \quad=\left\{\begin{array}{lll}
0 & & \text { if } F \nsubseteq T \\
\psi(T \backslash F) & \text { in } \mathbb{R}^{2 d} / \operatorname{span}(\psi(F)) & \text { if }\{u, v\} \subseteq F \subseteq T \\
(\psi(u)-\psi(v)) & \text { in } \mathbb{R}^{2 d} / \operatorname{span}(\psi(T \backslash\{u\})) & \text { if }\{u, v\} \subseteq T=F \uplus\{u\}(8) \\
-(\psi(u)-\psi(v)) & \text { in } \mathbb{R}^{2 d} / \operatorname{span}(\psi(T \backslash\{u\})) & \text { if }\{u, v\} \subseteq T=F \uplus\{v\} \\
\psi_{0}(T \backslash F) & \text { in } \mathbb{R}^{2 d} / \operatorname{span}\left(\psi_{0}(F)\right) & \text { otherwise. }
\end{array}\right.
\end{aligned}
$$

This follows directly from the definition of $\psi_{0}^{2 d}$ and the observation (7). Assume for a moment that $\psi_{0}^{2 d}$ is injective. Then for a small enough perturbation of the entries of a representing matrix of $\psi_{0}^{2 d}$, the columns of the resulted matrix would be independent, i.e. the corresponding linear transformation would be injective. In particular, there would exist an $\epsilon>0$ such that for every $0<t<\epsilon, \operatorname{Ker} \psi_{K, t}^{2 d}=0$. In particular, for a generic $\psi, \operatorname{Ker} \psi_{K}^{2 d}=0$. Thus, the following Lemma 5.2 completes the proof. 
Lemma $5.2 \psi_{0}^{2 d}$ is injective for a non-empty Zariski open set of maps $\psi$ : $K_{0} \longrightarrow \mathbb{R}^{2 d}$.

Proof For a linear transformation $C$, denote by $[C]$ its representing matrix w.r.t. given bases. In $\left[\psi_{0}^{2 d}\right]$ bases are indexed by sets according to (8) and each column represents the image $\psi_{0}^{2 d}(1 T)$ for some $T \in K_{d-1}$. Recall that for $T$ such that $\{u, v\} \subseteq T \in$ $K_{d-1},\left.\psi_{0}^{2 d}(T)\right|_{T \backslash v}=-\left.\psi_{0}^{2 d}(T)\right|_{T \backslash u}$.

First add rows indexed by $F^{\prime} \uplus\{u\}$ to rows $F^{\prime} \uplus\{v\}$ (in particular $F^{\prime} \cap\{u, v\}=\emptyset$ ), then delete the rows $F$ containing $u$, to obtain a matrix $[B]$, of a linear transformation $B$. In particular, we delete all rows $F$ such that $\{u, v\} \subseteq F$.

Note that $K_{0}^{\prime}=K_{0} \backslash\{u\}$, thus, for the obvious bases, $[B]$ is obtained from $\left[\left(\psi \mid K_{0}^{\prime}\right)_{K^{\prime}}^{2 d}\right]$ by doubling the columns indexed by $T^{\prime} \uplus\{v\} \in K_{d-1}^{\prime}$ where both $T^{\prime} \uplus$ $\{v\}, T^{\prime} \uplus\{u\} \in K_{d-1}$, and by adding a zero column for every $T^{\prime} \uplus\{u, v\} \in K_{d-1}$. For short, write $\psi_{K^{\prime}}^{2 d}=\left(\left.\psi\right|_{K_{0}^{\prime}}\right)_{K^{\prime}}^{2 d}$. More precisely, the linear maps $B$ and $\psi_{K^{\prime}}^{2 d}$ are related as follows: they have the same range. The domain of $B$ is $\operatorname{dom}(B)=\operatorname{dom}\left(\psi_{0}^{2 d}\right)=$ $D_{1} \oplus D_{2} \oplus D_{3}$ where

$D_{1}=\oplus\left\{\mathbb{R} T: T \in K_{d-1},\{u, v\} \nsubseteq T,(u \in T) \Rightarrow(T \backslash u) \cup v \notin K\right\}$,

$D_{2}=\oplus\left\{\mathbb{R} T: T \in K_{d-1}, u \in T, v \notin T,(T \backslash u) \cup v \in K\right\}$,

$D_{3}=\oplus\left\{\mathbb{R} T: T \in K_{d-1},\{u, v\} \subseteq T\right\}$.

For a base element $1 T$ of $D_{1}$, let $T^{\prime} \in K^{\prime}$ be obtained from $T$ by replacing $u$ with $v$. Then $B(1 T)=\psi_{K^{\prime}}^{2 d}\left(1 T^{\prime}\right)$; thus $\left.\operatorname{Ker} B\right|_{D_{1}} \cong \operatorname{Ker} \psi_{K^{\prime}}^{2 d}$. For a base element $1 T$ of $D_{2}$, $B(1 T)=\psi_{K^{\prime}}^{2 d}(1((T \backslash u) \cup v))$, and $\left.B\right|_{D_{3}}=0$.

Assume we have a linear dependency $\sum_{T \in K_{d-1}} \alpha_{T} \psi_{0}^{2 d}(T)=0$. By assumption, $\{d+2, d+3, \ldots, 2 d+1\} \notin \Delta^{s}\left(K^{\prime}\right)$, hence $\operatorname{Ker} \psi_{K^{\prime}}^{2 d}=0$. Thus, by inspecting the matrix $\left[\psi_{0}^{2 d}\right]$ and the matrix $[B]$ described above, we conclude that $\alpha_{T}=0$ for every base element $T$ except possibly for $T$ containing $\{u, v\}$ and for $T^{\prime} \uplus\{u\}, T^{\prime} \uplus\{v\} \in$ $K_{d-1}$, where $\alpha_{T^{\prime} \uplus\{u\}}=-\alpha_{T^{\prime} \uplus\{v\}}$. We need to show that $\alpha_{T^{\prime} \uplus\{v\}}=0$ for every $T^{\prime} \uplus$ $\{v\} \in K$ such that $T^{\prime} \cup\{u\} \in K$.

Let $\left.\psi_{0}^{2 d}\right|_{\text {res }}$ be the restriction of $\psi_{0}^{2 d}$ to the subspace spanned by the base elements $T=T^{\prime} \uplus\{v\}$ such that $T^{\prime} \cup\{u\} \in K$, followed by projection onto the subspace spanned by the $F \in\left(\begin{array}{c}K_{0} \\ d-1\end{array}\right)$ coordinates where $v \in F$ (just forget the other coordinates). As $\left.\psi_{0}^{2 d}(T)\right|_{F}=0$ whenever $F \ni v \notin T$, by inspecting the matrix $\left[\psi_{0}^{2 d}\right]$ restricted to rows $F$ with $v \in F$ and to columns $T=T^{\prime} \uplus\{v\}$ such that $T^{\prime} \cup\{u\} \in K$, we see that if $\left.\psi_{0}^{2 d}\right|_{\text {res }}$ is injective, then $\alpha_{T}=0$ for all $T=T^{\prime} \uplus\{v\} \in K$ such that $T^{\prime} \cup\{u\} \in K$. Thus, Lemma 5.3 below completes the proof.

Lemma $\left.5.3 \psi_{0}^{2 d}\right|_{\text {res }}$ is injective for a non-empty Zariski open set of maps $\psi$ : $K_{0} \longrightarrow \mathbb{R}^{2 d}$.

Proof Let $G=(\{u\} *(\operatorname{lk}(u, K) \cap \operatorname{lk}(v, K)))_{\leq d-2}$. Note that $v$ appears in the index set of every row and every column of $\left[\left.\psi_{0}^{2 d}\right|_{\text {res }}\right]$. Omitting $v$ from the indices of both of the bases used to define $\left.\psi_{0}^{2 d}\right|_{\text {res }}$, we notice that 


$$
\begin{aligned}
\left.\psi_{0}^{2 d}\right|_{\mathrm{res}} & \cong \overline{\left.\psi_{0}^{2 d}\right|_{\mathrm{res}}}: \oplus_{T \in G_{d-2}} \mathbb{R} T \longrightarrow \oplus_{F \in\left(\begin{array}{c}
G_{0} \\
d-2
\end{array}\right)} \mathbb{R}^{2 d} / \operatorname{span}(\psi(F \uplus\{v\})) \\
= & \oplus_{F \in\left(\begin{array}{c}
G_{0} \\
d-2
\end{array}\right)}\left(\mathbb{R}^{2 d} / \operatorname{span}(\psi(v))\right) / \overline{\operatorname{span}(\psi(F))}, \\
1 T & \mapsto \sum_{F \in\left(\begin{array}{c}
G_{0} \\
d-2
\end{array}\right)} \delta_{F \subseteq T} \overline{\psi(T \backslash F)} F
\end{aligned}
$$

where $\delta_{F \subseteq T}$ equals 1 if $F \subseteq T$ and 0 otherwise, and $\overline{\operatorname{span}(\psi(F))}$ is the image of $\operatorname{span}(\psi(F))$ in the quotient space $\mathbb{R}^{2 d} / \operatorname{span}(\psi(v))$.

Consider the projection $\pi: \mathbb{R}^{2 d} \longrightarrow \mathbb{R}^{2 d} / \operatorname{span}(\psi(v)) \cong \mathbb{R}^{2 d-1}$. Let $\bar{\psi}=\pi \circ$ $\left.\psi\right|_{G_{0}}: G_{0} \longrightarrow \mathbb{R}^{2 d-1}$, and $\bar{\psi}_{G}^{2 d-1}$ be the induced map as defined in (6). Then $\pi$ induces $\pi_{*} \overline{\left.\psi_{0}^{2 d}\right|_{\text {res }}}=\bar{\psi}_{G}^{2 d-1}$.

By assumption, $\{d+1, \ldots, 2 d-1\} \notin \Delta^{s}(\operatorname{lk}(u, K) \cap \operatorname{lk}(v, K))$. As symmetric shifting commutes with constructing a cone (Kalai [11, Theorem 2.2.8], and Babson, Novik and Thomas [1, Theorem 3.7]), $\{d+2, \ldots, 2 d\} \notin \Delta^{s}(G)$. Hence $y_{2 d}^{d-1} \notin \mathrm{GIN}(G)$, and by Lee [13], $\operatorname{Ker} \phi_{G}^{2 d-1}=0$ for a generic $\phi$. Thus, all liftings $\psi: K_{0} \longrightarrow \mathbb{R}^{2 d}$ such that $\bar{\psi}=\phi$ satisfy $\left.\operatorname{Ker} \psi_{0}^{2 d}\right|_{\text {res }} \cong \operatorname{Ker} \phi_{G}^{2 d-1}=0$, and this set of liftings is a non-empty Zariski open set.

Clearly the set of all $\psi$ such that $\psi_{K}^{2 d}$ is injective is Zariski open. We exhibited conditions under which it is non-empty.

Proof for exterior shifting The proof is similar to the proof for the symmetric case. We indicate the differences. The map $\psi: K_{0} \rightarrow \mathbb{R}^{d+1}$ defines the first $d+1$ generic $f_{i}$ 's w.r.t. the $e_{i}$ 's basis of $\mathbb{R}^{\left|K_{0}\right|}$ and induces the following map:

$$
\psi_{K, \mathrm{ext}}^{d+1}: \oplus_{T \in K_{d-1}} \mathbb{R} T \longrightarrow \oplus_{1 \leq i \leq d+1} \oplus_{F \in\left(\begin{array}{c}
K_{0} \\
d-1
\end{array}\right)} \mathbb{R} F, m \mapsto\left(f _ { 1 } \left\lfloorm, \ldots, f_{d+1}\lfloor m)\right.\right.
$$

where $f_{i}\left\lfloor\right.$. is the left interior product given by bilinear extension of $e_{S}\left\lfloor e_{T}=\right.$ $\delta_{S \subseteq T} \operatorname{sign}(S, T) e_{T \backslash S}$, as in [8]. By [20, Proposition 3.1],

$\operatorname{Ker} \psi_{K, \text { ext }}^{d+1}=\cap_{1 \leq i \leq d+1} \operatorname{Ker} f_{i}\left\lfloor=\cap_{R<\text { lex }}\{d+2, \ldots, 2 d+1\} \operatorname{Ker}\left(f_{R}\left\lfloor: \oplus_{T \in K_{d-1}} \mathbb{R} T \longrightarrow \mathbb{R}\right)\right.\right.$, and hence by shiftedness $\{d+2, \ldots, 2 d+1\} \notin \Delta^{e}(K) \Leftrightarrow \operatorname{Ker} \psi_{K \text {,ext }}^{d+1}=0$.

Replacing $\psi(u)$ by $\psi(v)$ induces a map

$$
\psi_{K, u}^{d+1}: \oplus_{T \in K_{d-1}} \mathbb{R} T \longrightarrow \bigoplus_{1 \leq i \leq d+1} \oplus_{F \in\left(\begin{array}{c}
K_{0} \\
d-1
\end{array}\right)} \mathbb{R} F
$$

Note that if $\operatorname{Ker} \psi_{K, u}^{d+1}=0$ then $\operatorname{Ker} \psi_{K, \text { ext }}^{d+1}=0$ for generic $\psi$. Let [ $B_{\text {ext }}$ ] be obtained from the matrix $\left[\psi_{K, u}^{d+1}\right]$ by adding the rows indexed by $F^{\prime} \uplus u$ to the corresponding rows indexed by $F^{\prime} \uplus v$ and deleting the rows indexed by $F$ with $u \in F$. The domain of $B_{\text {ext }}$ is $D_{1} \oplus D_{2} \oplus D_{3}$ defined by sets indexing a basis as for $B$ in the symmetric case. For a base element $1 T$ of $D_{1}$, let $T^{\prime} \in K^{\prime}$ be obtained from $T$ by replacing $u$ with $v$. Then $B_{\text {ext }}(1 T)=\psi_{K^{\prime} \text {,ext }}^{d+1}\left(1 T^{\prime}\right)$; thus $\left.\operatorname{Ker} B_{\text {ext }}\right|_{D_{1}} \cong \operatorname{Ker} \psi_{K^{\prime} \text {,ext }}^{d+1}$. For a base element $1 T$ of $D_{2}, B_{\text {ext }}(1 T)=\psi_{K^{\prime}, \text { ext }}^{d+1}(1((T \backslash u) \cup v))$, and as we may number $v=1$, 
$u=2$ then $\left.B\right|_{D_{3}}=0$ (for each column indexed by $F^{\prime} \uplus\{u, v\}$, the rows of $F^{\prime} \uplus u$ and of $F^{\prime} \uplus v$ have opposite signs in $\psi_{K, u}^{d+1}$ ). Now we can adopt the arguments showing that $\operatorname{Ker} \psi_{0}^{2 d}=0$ using $B$ in the symmetric case, to show that $\operatorname{Ker} \psi_{K, u}^{d+1}=0$ using $B_{\text {ext }}$. This settles the case $\mathbb{F}=\mathbb{R}$.

As $\Delta^{e}(K)$ is shifted when defined w.r.t. any field, the proof above holds for any infinite field.

Recall that the $d$-Lefschetz property for a $2 d$-homology sphere $K$ is equivalent to $y_{2 d+3}^{d+1} \notin \mathrm{GIN}(K)$ (for a generic basis $\left\{y_{1}, \ldots, y_{n}\right\}$ w.r.t $\left\{x_{1}, \ldots, x_{n}\right\}$ ), which in turn is equivalent to $\{d+3, \ldots, 2 d+3\} \notin \Delta^{s}(K)$. We conclude the following.

Corollary 5.4 Let $K$ be a $2 d$-sphere for some $d \geq 1$, and let $a, b \in K$ be two vertices which satisfy the Link Condition, i.e. such that $\operatorname{lk}(a, K) \cap \operatorname{lk}(b, K)=\operatorname{lk}(\{a, b\}, K)$. Let $K^{\prime}$ be obtained from $K$ by contracting $a \mapsto b$. Then:

(1) $K^{\prime}$ is a $2 d$-sphere, PL homeomorphic to $K$ ([21, Theorem 1.4]).

(2) If $K^{\prime}$ is $d$-Lefschetz and $\operatorname{lk}(\{a, b\}, K)$ is $(d-1)$-Lefschetz over $\mathbb{R}$, then $K$ is $d$-Lefschetz over $\mathbb{R}$ (by Proposition 5.1).

Let $K$ be a simplicial complex. Its Stellar subdivision at a face $T \in K$ is the operation $K \mapsto K^{\prime}$ where

$$
K^{\prime}=\operatorname{Stellar}(T, K):=(K \backslash \operatorname{st}(T, K)) \cup\left(\left\{v_{T}\right\} * \partial T * \operatorname{lk}(T, K)\right),
$$

where $v_{T}$ is a vertex not in $K$ and $\operatorname{st}(T, K)=\{S \in K: T \subseteq S\}$. Note that for $u \in$ $T \in K, u, v_{T} \in K^{\prime}$ satisfy the Link Condition and their identification results in $K$. Further, $\operatorname{lk}\left(\left\{u, v_{T}\right\}, K^{\prime}\right)=\operatorname{lk}(u, \partial T * \operatorname{lk}(T, K))=\partial(T \backslash\{u\}) * \operatorname{lk}(T, K)$.

Proof of Theorem 1.2(3) Let $T=\operatorname{Stellar}(F, K)$, denote its dimension by $d-1$, and assume by contradiction that $T$ is not SL. As we have seen in the proof of Lemma 4.3 , there exists $0 \leq i_{0} \leq\lfloor d / 2\rfloor$ such that $\Omega_{S L}\left(T, i_{0}\right)=\emptyset$. First we show that $i_{0} \neq$ $\lfloor d / 2\rfloor$ : for even $d$ this is obvious. For odd $d$, note that for $u \in F$ the contraction $v_{F} \mapsto u$ in $T$ results in $K$, which is $\lfloor d / 2\rfloor$-Lefschetz. Further, the $(d-3)$-sphere $\operatorname{lk}\left(\left\{v_{F}, u\right\}, T\right)=\operatorname{lk}(F, K) * \partial(F \backslash\{u\})$ is SL by Theorem 1.2(1), and in particular is $(\lfloor d / 2\rfloor-1)$-Lefschetz. Thus, by Corollary 5.4, $T$ is $\lfloor d / 2\rfloor$-Lefschetz, and hence $0 \leq i_{0} \leq\lfloor d / 2\rfloor-1$.

Let $L=T * \partial \sigma$, where $\sigma$ is the $\left(d-2 i_{0}-1\right)$-simplex (then $L$ has even dimension $\left.2 d-2 i_{0}-2\right)$. By Lemma 4.3, for any two 1.s.o.p.'s $\Theta_{T}$ and $\Theta_{\partial \sigma}$ of $\mathbb{R}[T]$ and $\mathbb{R}[\partial \sigma]$ respectively, $\mathbb{R}[L] /\left(\Theta_{T} \cup \Theta_{\partial \sigma}\right)$ has no $\left(d-i_{0}-1\right)$-Lefschetz element.

On the other hand, we shall now prove the existence of such 1.s.o.p.'s and a $(d-$ $\left.i_{0}-1\right)$-Lefschetz element, to reach a contradiction. This requires a close look at the proof of Proposition 5.1.

Note that $\mathbb{R}[L] /\left(\Theta_{T} \cup \Theta_{\partial \sigma}\right)$ has a $\left(d-i_{0}-1\right)$-Lefschetz element for some 1.s.o.p.'s $\Theta_{T}, \Theta_{\partial \sigma}$ of $T$ and $\partial \sigma$ respectively, iff for some map $\psi: L_{0} \rightarrow \mathbb{R}^{d} \oplus$ $\mathbb{R}^{d-2 i_{0}-1} \oplus \mathbb{R}$ such that $\psi\left(T_{0}\right) \subseteq \mathbb{R}^{d} \oplus 0 \oplus \mathbb{R}$ and $\psi\left(\sigma_{0}\right) \subseteq 0 \oplus \mathbb{R}^{d-2 i_{0}-1} \oplus \mathbb{R}$, the kernel $\operatorname{Ker}\left(\psi_{L}^{2 d-2 i_{0}}\right)$ vanishes. Here we identify $\psi$ with linear forms in $\mathbb{R}[L]$ such that the first $d$ columns in [ $\psi]$ correspond to $\Theta_{T}$, the next $d-2 i_{0}-1$ columns correspond to $\Theta_{\partial \sigma}$ and the last column correspond to a $\left(d-i_{0}-1\right)$-Lefschetz element $\omega$. 


\begin{tabular}{|c|c|c|c|c|c|}
\hline$W$ & \multicolumn{3}{|c|}{$\left|\Theta_{T}\right| \Theta_{\partial \sigma}|\omega|$} & \multicolumn{2}{|c|}{$W_{0} \| \Theta_{\partial \sigma}|\omega|$} \\
\hline$v_{F}$ & $*$ & 0 & $*$ & 0 & 0 \\
\hline$u$ & $*$ & 0 & $*$ & 0 & 0 \\
\hline $\operatorname{lk}\left(\left\{v_{F}, u\right\}, L\right)_{0}$ & $*$ & 0 & $*$ & 0 & 0 \\
\hline$L_{0} \backslash\left(\operatorname{lk}\left(\left\{v_{F}, u\right\}, L\right)_{0} \cup\left\{v_{F}, u\right\}\right)$ & * & 0 & $*$ & 0 & 0 \\
\hline$\sigma_{0}$ & 0 & $*$ & $*$ & $*$ & * \\
\hline
\end{tabular}

\begin{tabular}{|c|c|c|c|c|c|}
\hline$W_{1} \| \Theta_{T}$ & & $W_{2} \| \Theta_{T} \backslash$ & $\Theta_{\partial \sigma}$ & $\omega$ & $W_{3} \| \Theta_{T}$ \\
\hline 0 & 0 & 0 & 0 & 0 & $*$ \\
\hline$*$ & * & * & 0 & $*$ & $*$ \\
\hline$*$ & $*$ & $*$ & 0 & $*$ & $*$ \\
\hline$*$ & $*$ & 0 & 0 & 0 & $*$ \\
\hline 0 & 0 & 0 & $*$ & $*$ & 0 \\
\hline
\end{tabular}

Fig. 1 The spaces $W, W_{0}, W_{1}, W_{2}, W_{3}$. (Same row labels for all, written explicitly for $W$ and left blank for the rest. The $*$ stands for arbitrary $\mathbb{R}$-coordinates)

Indeed, such a generic block matrix on the first $2 d-2 i_{0}-1$ columns induces two 1.s.o.p.'s, for $\mathbb{R}[T]$ and $\mathbb{R}[\partial \sigma]$, whose union is an l.s.o.p. for $\mathbb{R}[L]$ by Theorem 2.2 , and for the statement about the $\left(d-i_{0}-1\right)$-Lefschetz element we use the results in [13] and [26] as in the proof of Proposition 5.1.

Note that $L=\operatorname{Stellar}(F, K * \partial \sigma)$, and that for $u \in F$ the contraction $v_{F} \mapsto u$ in $L$ results in $K * \partial \sigma$. Further, $\operatorname{lk}\left(\left\{v_{F}, u\right\}, L\right)=\operatorname{lk}(F, K) * \partial(F \backslash\{u\}) * \partial \sigma$.

We now show that there exists a map $\psi: L_{0} \longrightarrow \mathbb{R}^{d} \oplus \mathbb{R}^{d-2 i_{0}-1} \oplus \mathbb{R}$ for which the following four properties hold simultaneously:

(0) $\psi\left(\sigma_{0}\right) \subseteq 0 \oplus \mathbb{R}^{d-2 i_{0}-1} \oplus \mathbb{R}$ and induces an 1.s.o.p. $\Theta_{\partial \sigma}$ of $\mathbb{R}[\partial \sigma]$ and an SL element $\omega_{\partial \sigma}$ of $\mathbb{R}[K] /\left(\Theta_{\partial \sigma}\right)$ (by last $d-2 i_{0}$ columns).

(1) $\psi\left(K_{0}\right) \subseteq \mathbb{R}^{d} \oplus 0 \oplus \mathbb{R}$ and induces an l.s.o.p. $\Theta_{K}$ of $\mathbb{R}[K]$ (by first $d$ columns) and an SL element $\omega_{K}$ of $\mathbb{R}[K] /\left(\Theta_{K}\right)$ (by last column).

(2) $0 \neq \psi\left(v_{F}\right) \in \mathbb{R}^{d} \oplus 0 \oplus \mathbb{R}$ induces a map $\pi: \mathbb{R}^{2 d-2 i_{0}} \rightarrow \mathbb{R}^{2 d-2 i_{0}} / \operatorname{span} \psi\left(v_{F}\right) \cong$ $\mathbb{R}^{2 d-2 i_{0}-1}$ such that $\left.\pi \circ \psi\right|_{1 \mathrm{k}(F, K)_{0} \cup F_{0} \cup \sigma_{0}}$ induces an element in $\Omega\left(G, d-i_{0}-2\right)$ for $G=\{u\} * \operatorname{lk}\left(\left\{v_{F}, u\right\}, L\right)$.

(3) $\psi\left(K_{0} \cup\left\{v_{F}\right\}\right) \subseteq \mathbb{R}^{d} \oplus 0 \oplus \mathbb{R}$ and the first $d$ columns of [ $\left.\psi\right]$ induce an 1.s.o.p. $\Theta_{T}$ of $\mathbb{R}[T]$.

To see this, we show that in the linear space $W$ of maps $\left\{f: L_{0} \rightarrow \mathbb{R}^{2 d-2 i_{0}}\right\} \cong$ $\mathbb{R}^{\left|L_{0}\right| \times\left(2 d-2 i_{0}\right)}$ such that $\psi\left(T_{0}\right) \subseteq \mathbb{R}^{d} \oplus 0 \oplus \mathbb{R}$ and $\psi\left(\sigma_{0}\right) \subseteq 0 \oplus \mathbb{R}^{d-2 i_{0}-1} \oplus \mathbb{R}$, the Zariski open set $\Omega$ of maps for which properties (0),(1),(2) and (3) hold is nonempty. We will look at projections onto linear spaces $\pi_{i}: W \rightarrow W_{i}$, the $W_{i}$ 's are defined in Fig. 1, and at Zariski nonempty open sets $\Omega_{i} \subseteq W_{i}$ such that an element in $\pi_{i}^{-1}\left(\Omega_{i}\right)$ has property (i) for $i=0,1,2,3$. The intersection of these four nonempty Zariski open sets is nonempty, hence it will follow that $\Omega$ is nonempty. See Figure 1 for illustration. 
As $T$ is CM, $\Omega_{3} \neq \emptyset$, corresponding to 1.s.o.p.'s of $\mathbb{R}[T]$. As $K$ is SL, $\Omega_{1} \neq \emptyset$, corresponding to pairs of 1.s.o.p. and an SL element of $\mathbb{R}[K]$. As $\partial \sigma$ is SL, $\Omega_{0} \neq \emptyset$, corresponding to pairs of 1.s.o.p. and an SL element of $\mathbb{R}[\partial \sigma]$. By Theorem 2.2,

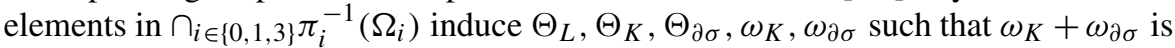
an SL element of $\mathbb{R}[K * \partial \sigma] /\left(\Theta_{K} \cup \Theta_{\partial \sigma}\right)$, and $\Theta_{T} \cup \Theta_{\partial \sigma}$ is an 1.s.o.p. of $\mathbb{R}[L]$.

To show property (2), first note that by Theorem 2.2 and the assumption that $\operatorname{lk}(F, K)$ is $\mathrm{SL}$, there is a pair $(\Theta, \omega)$ of an 1.s.o.p. and an SL element for $\operatorname{lk}\left(\left\{v_{F}, u\right\}, L\right)=\operatorname{lk}(F, K) * \partial(F \backslash\{u\}) * \partial \sigma$ such that $\Theta=\Theta_{\operatorname{lk}(F, K)} \uplus \Theta_{\partial(F \backslash\{u\})} \uplus \Theta_{\partial \sigma}$ for 1.s.o.p's of the corresponding complexes. By adding $x_{u}$ to this 1.s.o.p. we obtain an l.s.o.p. for $G$ where $\omega: H(G)_{d-i_{0}-2} \rightarrow H(G)_{d-i_{0}-1}$ is injective; hence $\Omega_{2} \neq \emptyset$. Thus, a generic element in $W$ satisfies property (2).

To summarize, we showed that $\Omega$ is nonempty. Let $\psi \in \Omega$ and define for $0<t \leq 1$ the map $\psi_{t}: L_{0} \rightarrow \mathbb{R}^{2 d-2 i_{0}}$ by $\psi_{t}\left(v_{F}\right)=\psi(u)+t\left(\psi\left(v_{F}\right)-\psi(u)\right)$ and $\psi_{t}(v)=$ $\psi(v)$ for any $v_{F} \neq v \in L_{0}$. As before, define $\psi_{0}^{2 d-2 i_{0}}=\lim _{t \mapsto 0} \psi_{L, t}^{2 d-2 i_{0}}$, see (8). Now repeat the proof of Proposition 5.1 w.r.t. $\psi_{0}^{2 d-2 i_{0}}$. Thus, for a small enough $\epsilon$, the map $\psi^{\prime}: L_{0} \longrightarrow \mathbb{R}^{2 d-2 i_{0}}$ defined by $\psi^{\prime}\left(v_{F}\right)=\psi(u)+\epsilon\left(\psi\left(v_{F}\right)-\psi(u)\right)$ and $\psi^{\prime}(v)=\psi(v)$ for every other vertex $v \in L_{0}$, satisfies $\operatorname{Ker}\left(\psi^{\prime}\right)_{L}^{2 d-2 i_{0}}=0$. Thus, the first $d$ columns of $\left[\psi^{\prime}\right]$ induce an 1.s.o.p. $\Theta_{T}$ of $T$, the next $d-i_{0}-1$ columns induce an l.s.o.p. $\Theta_{\partial \sigma}$ of $\partial \sigma$, and the last column of $\left[\psi^{\prime}\right]$ is a $\left(d-i_{0}-1\right)$-Lefschetz element of $\mathbb{R}[L] /\left(\Theta_{T} \cup \Theta_{\partial \sigma}\right)$. This contradicts our earlier conclusion, which was based on assuming that the assertion of this theorem is incorrect.

Corollary 5.5 Let $\mathcal{S}$ be a family of homology spheres which is closed under taking links and such that all of its elements are $S L$, over $\mathbb{R}$. Let $\mathbb{S}=\mathbb{S}(\mathcal{S})$ be the family obtained from $\mathcal{S} \cup\left\{\partial \sigma^{n}: n \geq 1\right\}$ by taking the closure under the operations of: (0) taking links; (1) join; (2) Stellar subdivisions. Then every element in $\mathbb{S}$ is $S L$.

Proof We prove by double induction - on dimension, and on the sequence of operations of types (0), (1) and (2) which define $S \in \mathbb{S}$ - that $S$ and all its face links are SL. Let us call $S$ with this property hereditary $S L$.

Note that every $S \in \mathcal{S}$ and every boundary of a simplex, is hereditary SL. This includes the (unique) zero-dimensional sphere and provides the base of the induction. (Actually it is known that every (homology) sphere of dimension $\leq 2$ is hereditary SL.)

Clearly if $S$ is hereditary SL, then so are all of its links, as $\operatorname{lk}(Q,(\operatorname{lk}(F, S)))=$ $\operatorname{lk}(Q \uplus F, S)$. If $S$ and $S^{\prime}$ are hereditary SL then by Theorem 2.2 so is $S * S^{\prime}$ (here we note that every $T \in S * S^{\prime}$ is of the form $T=F \uplus F^{\prime}$ where $F \in S$ and $F^{\prime} \in S^{\prime}$, and that $\left.\operatorname{lk}\left(T, S * S^{\prime}\right)=\operatorname{lk}(F, S) * \operatorname{lk}\left(F^{\prime}, S^{\prime}\right)\right)$. We are left to show that if $F \in S$ and $S$ is hereditary SL, then so is $T:=\operatorname{Stellar}(F, S)$. Assume $\operatorname{dim} F \geq 1$, otherwise there is nothing to prove. First we note that by the induction hypothesis for every $v \in T_{0}$, $\operatorname{lk}(v, T)$ is hereditary SL:

Case 1: $v=v_{F}$. Then $\operatorname{lk}\left(v_{F}, T\right)=\operatorname{lk}(F, S) * \partial F$ is hereditary SL by Theorem 2.2, as argued above.

Case 2: $v \in F$. Then $\operatorname{lk}(v, T)=\operatorname{Stellar}(F \backslash\{v\}, \operatorname{lk}(v, S))$ is hereditary SL by the induction hypothesis on the dimension.

Case 3: $v \notin F, v \neq v_{F}$ and $F \in \operatorname{lk}(v, S)$. Then $\operatorname{lk}(v, T)=\operatorname{Stellar}(F, \operatorname{lk}(v, S))$ is 
hereditary SL by the induction hypothesis on the dimension.

Otherwise: $\operatorname{lk}(v, T)=\operatorname{lk}(v, S)$ is hereditary SL.

We are left to show that $T$ is SL: $S$ is SL, and for $u \in F, \operatorname{lk}\left(\left\{v_{F}, u\right\}, T\right)=$ $\operatorname{lk}(F, S) * \partial(F \backslash\{u\})$ is SL by Theorem 2.2. Thus, by Theorem 1.2(3) $T$ is SL, and together with the above, $T$ is hereditary SL.

The barycentric subdivision of a simplicial complex $K$ can be obtained by a sequence of Stellar subdivisions: order the faces of $K$ of dimension $>0$ by weakly decreasing size, and perform Stellar subdivisions at those faces according to this order; the barycentric subdivision of $K$ is obtained. Brenti and Welker [4, Corollary 3.5] showed that the $h$-polynomial of the barycentric subdivision of a Cohen-Macaulay complex has only simple and real roots, and hence is unimodal. In particular, barycentric subdivision preserves non-negativity of the $g$-vector for spheres all of whose links are SL. The above corollary shows that the hereditary SL property itself is preserved.

Recently Martina Kubitzke and the second author showed that the $g$-vector of the barycentric subdivision of a CM complex is an $M$-sequence [12].

\section{Lefschetz properties and connected sum}

Let $K$ and $L$ be pure simplicial complexes which intersect in a common closed facet $\langle\sigma\rangle=K \cap L$. Their connected sum over $\sigma$ is $K \#_{\sigma} L=(K \cup L) \backslash\{\sigma\}$.

Theorem 6.1 Let $K$ and $L$ be homology $(d-1)$-spheres over an infinite field $\mathbb{F}$ which intersect in a common closed facet $\langle\sigma\rangle=K \cap L$. Let $A=\mathbb{F}\left[x_{v}: v \in(K \cup L)_{0}\right]$. Then:

(0) $K \#_{\sigma} L$ is a homology $(d-1)$-sphere; in particular its h-vector is symmetric.

(1) Let $\Theta$ be a common l.s.o.p for $K, L,\langle\sigma\rangle$ and $K \#_{\sigma} L$ over $A$ (it exists if $\mathbb{F}$ is infinite). Assume that $K$ and $L$ are $i$-Lefschetz for some $i>0$ and let $\omega$ be an $i$-Lefschetz element for both $K$ and $L$ w.r.t. $\Theta$ (it exists). Then $\omega$ is an $i$-Lefschetz element of $\mathbb{F}\left[K \#_{\sigma} L\right] /(\Theta)$.

Proof Straightforward Mayer-Vietoris and Euler characteristic arguments show that $K \#_{\sigma} L$ is a homology $(d-1)$-sphere.

For a simplicial complex $M$ let $\mathbb{F}(M):=\bigoplus_{a: \operatorname{supp}(a) \in M} \mathbb{F} x^{a}$ be a module over $A_{M_{0}}=\mathbb{F}\left[x_{v}: v \in M_{0}\right]$ defined by

$$
x_{v}\left(x^{a}\right)= \begin{cases}x_{v} x^{a} & \text { if } v \cup \operatorname{supp}(a) \in M, \\ 0 & \text { otherwise. }\end{cases}
$$

Note that $\mathbb{F}(M) \cong \mathbb{F}[M]$ as $A_{M_{0}}$-modules. For $v \in(K \cup L)_{0} \backslash L_{0}$ and $m \in \mathbb{F}(L)$, $x_{v} m=0$. Then the following is an exact sequence of $A$-modules:

$$
0 \rightarrow \mathbb{F}(\langle\sigma\rangle) \stackrel{(\iota,-\iota)}{\longrightarrow}(\mathbb{F}(K) \oplus \mathbb{F}(L)) \stackrel{\iota_{K}+\iota_{L}}{\longrightarrow} \mathbb{F}\left(K \cup_{\sigma} L\right) \rightarrow 0
$$

where the 's denote the obvious inclusions. As $\mathbb{F}$ is infinite, there exists a 1.s.o.p. for each of the $(d-1)$-complexes in Theorem 6.1(1), and as a finite intersection of Zariski nonempty open sets is nonempty, $\Theta$ as in (1) exists. When we mod out (10) 
by $\Theta$, which is the same as to tensor (10) with $\otimes_{A} A / \Theta$, we obtain an exact sequence of $A$-modules:

$$
\frac{\mathbb{F}(\langle\sigma\rangle)}{(\Theta) \mathbb{F}(\langle\sigma\rangle)} \rightarrow \frac{\mathbb{F}(K)}{(\Theta) \mathbb{F}(K)} \oplus \frac{\mathbb{F}(L)}{(\Theta) \mathbb{F}(L)} \rightarrow \frac{\mathbb{F}\left(K \cup_{\sigma} L\right)}{(\Theta) \mathbb{F}\left(K \cup_{\sigma} L\right)} \rightarrow 0
$$

where in the middle term we used distributivity of $\otimes$ over $\oplus$. Note that $\frac{\mathbb{F}(\langle\sigma\rangle)}{(\Theta) \mathbb{F}(\langle\sigma\rangle)} \cong$ $\mathbb{F}$ is concentrated in degree 0 and that $\left(\mathbb{F}\left(K \#_{\sigma} L\right) /(\Theta)\right)_{<d} \cong\left(\mathbb{F}\left(K \cup_{\sigma} L\right) /(\Theta)\right)_{<d}$. Thus, for $0<i \leq d / 2$ we obtain the following commutative diagram of $A$-modules:

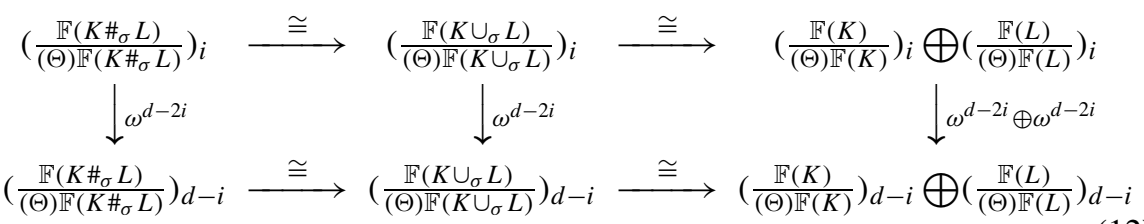

where the right vertical arrow is an isomorphism by our assumption. Hence, the left vertical arrow is an isomorphism as well, meaning that $\omega$ is an $i$-Lefschetz element of $\mathbb{F}\left[K \#_{\sigma} L\right] /(\Theta)$.

Proof of Theorem 1.2(2) If $K$ and $L$ are SL homology $(d-1)$-spheres then by Theorem $6.1 K \# L$ is a homology $(d-1)$-sphere and has a pair $(\Theta, \omega)$ of 1.s.o.p. and $i$-Lefschetz element for every $0<i \leq\lfloor d / 2\rfloor$.

For $i=0$, as $K \# L$ is Cohen-Macaulay with 1.s.o.p. $\Theta$ and $h_{d}=1$, then there exists a 0 -Lefschetz element $\tilde{\omega}$, i.e. $\tilde{\omega}^{d} \neq 0$. (This is equivalent to $[2, d+1] \in \Delta^{s}(K \# L)$, which reflects the fact that $K \# L$ has non-vanishing top homology.) By Lemma 4.1 the sets of 0 -Lefschetz elements and of $(0<)$-Lefschetz elements are Zariski open. The fact that they are nonempty implies that so is their intersection, i.e. $K \# L$ is SL. Similarly, one concludes that if $K$ and $L$ are weak-Lefschetz then so is $K \# L$.

Remark 6.2 The assertion of Theorem 1.2(2), rephrased in terms of algebraic shifting, says that if $\Delta^{s}(K), \Delta^{s}(L) \subseteq \Delta(d)$ then also $\Delta^{s}(K \# L) \subseteq \Delta(d)$. The analogous statement for exterior shifting is also true. These assertions follow from the characterization of the algebraic shifting of a union of complexes whose intersection is a simplex, given in [20]. To obtain the shifting of $K \# L$ from the shifting of $K \cup L$ just delete the facet $\{2,3, \ldots, d, d+2\}$ which represent the extra top homology in $K \cup L$.

Acknowledgements We deeply thank Satoshi Murai for his valuable comments on an earlier version of this paper, and Gil Kalai for helpful discussions. Further thanks go to the referees, especially one of them, whose detailed comments greatly helped to improve the presentation. Part of this work was done during the "Algebraic Combinatorics" program at the Institut Mittag-Leffler in Spring 2005. We are grateful to Anders Björner and Richard Stanley for inviting us to this program. The authors were partially supported by the European Commission's IHRP Programme, grant HPRN-CT-2001-00272, "Algebraic Combinatorics in Europe". Another part of this work was done during the special semester at the Institute for Advanced Studies in Jerusalem, in Spring 2007. We are grateful to IAS for the hospitality, and to Gil Kalai for organizing this semester. 


\section{References}

1. Babson, E., Novik, I., Thomas, R.: Reverse lexicographic and lexicographic shifting. J. Algebr. Comb. 23(2), 107-123 (2006). MR MR2223682 (2007a:13026)

2. Björner, A., Kalai, G.: An extended Euler-Poincaré theorem. Acta Math. 161(3-4), 279-303 (1988). MR MR971798 (89m:52009)

3. Björner, A., Kalai, G.: On $f$-vectors and homology. In: Combinatorial Mathematics: Proceedings of the Third International Conference, New York, 1985. Ann. New York Acad. Sci., vol. 555, pp. 63-80. New York Acad. Sci., New York (1989). MR MR1018610 (90i:52008)

4. Brenti, F., Welker, V.: $f$-vectors of barycentric subdivisions. Math. Z. 259(4), 849-865 (2008). MR MR2403744

5. Harima, T., Migliore, J.C., Nagel, U., Watanabe, J.: The weak and strong Lefschetz properties for Artinian $K$-algebras. J. Algebra 262(1), 99-126 (2003). MR MR1970804 (2004b:13001)

6. Harima, T., Watanabe, J.: The finite free extension of Artinian $K$-algebras with the strong Lefschetz property. Rend. Sem. Mat. Univ. Padova 110, 119-146 (2003). MR MR2033004 (2005a:13002)

7. Kalai, G.: Characterization of $f$-vectors of families of convex sets in $\mathbf{R}^{d}$. I. Necessity of Eckhoff's conditions. Israel J. Math. 48(2-3), 175-195 (1984). MR MR770700 (86c:52003)

8. Kalai, G.: Hyperconnectivity of graphs. Graphs Comb. 1(1), 65-79 (1985). MR MR796184 (87c:05081)

9. Kalai, G.: Symmetric matroids. J. Comb. Theory Ser. B 50(1), 54-64 (1990). MR MR1070465 (91m:05053)

10. Kalai, G.: The diameter of graphs of convex polytopes and $f$-vector theory. In: Applied Geometry and Discrete Mathematics. DIMACS Ser. Discrete Math. Theoret. Comput. Sci., vol. 4, pp. 387-411. Amer. Math. Soc., Providence (1991)

11. Kalai, G.: Algebraic shifting. In: Computational Commutative Algebra and Combinatorics, Osaka, 1999. Adv. Stud. Pure Math., vol. 33, pp. 121-163. Math. Soc. Japan, Tokyo (2002). MR MR1890098 (2003e:52024)

12. Kubitzke, M., Nevo, E.: The Lefschetz property for barycentric subdivisions of shellable complexes. To appear in Trans. Amer. Math. Soc. Math. arXiv:0712.1560 (2007)

13. Lee, C.W.: Generalized stress and motions. In: Polytopes: Abstract, Convex and Computational, Scarborough, ON, 1993. NATO Adv. Sci. Inst. Ser. C Math. Phys. Sci., vol. 440, pp. 249-271. Kluwer Acad. Publ., Dordrecht (1994). MR MR1322066 (96c:52039)

14. Lickorish, W.B.R.: Simplicial moves on complexes and manifolds. In: Proceedings of the Kirbyfest, Berkeley, CA, 1998. Geom. Topol. Monogr., vol. 2, pp. 299-320. Geom. Topol. Publ., Coventry (1999) (electronic)

15. McMullen, P.: The numbers of faces of simplicial polytopes. Israel J. Math. 9, 559-570 (1971). MR MR0278183 (43 \#3914)

16. McMullen, P.: Weights on polytopes. Discrete Comput. Geom. 15(4), 363-388 (1996). MR MR1384882 (97b:52012)

17. McMullen, P.: On simple polytopes. Invent. Math. 113(2), 419-444 (1993). MR MR1228132 (94d:52015)

18. Murai, S.: Algebraic shifting of cyclic polytopes and stacked polytopes. Discrete Math. 307(14), 1707-1721 (2007). MR MR2316811

19. Murai, S.: Algebraic shifting of strongly edge decomposable spheres. Math. arXiv:0709.4518 (2007)

20. Nevo, E.: Algebraic shifting and basic constructions on simplicial complexes. J. Algebraic Combin. 22(4), 411-433 (2005). MR MR2191645 (2006h:05239)

21. Nevo, E.: Higher minors and Van Kampen's obstruction. Math. Scandi. 101, 161-176 (2007)

22. Stanley, R.P.: The number of faces of a simplicial convex polytope. Adv. Math. 35(2), 236-238 (1980). MR MR563925 (81f:52014)

23. Stanley, R.P.: Combinatorics and commutative algebra, 2nd edn. Progress in Mathematics, vol. 41. Birkhäuser Boston, Boston (1996). MR MR1453579 (98h:05001)

24. Swartz, E.: $g$-elements, finite buildings and higher Cohen-Macaulay connectivity. J. Comb. Theory Ser. A 113(7), 1305-1320 (2006). MR MR2259062

25. Swartz, E.: Face enumeration—from spheres to manifolds. Math. arXiv:0709.3998 (2007)

26. Tay, T.-S., White, N., Whiteley, W.: Skeletal rigidity of simplicial complexes I. Eur. J. Comb. 16(4), 381-403 (1995). MR MR1337142 (96e:52052)

27. Whiteley, W.: Vertex splitting in isostatic frameworks. Struc. Top. 16, 23-30 (1989) 